\title{
Granular Structure Determined by Terahertz Scattering
}

\author{
Philip Born, ${ }^{1}$ Nick Rothbart, ${ }^{2,3}$ Matthias Sperl, ${ }^{1}$ and Heinz-Wilhelm Hübers ${ }^{2,3}$ \\ ${ }^{1}$ Institut für Materialphysik im Weltraum, Deutsches Zentrum für Luft- und Raumfahrt, 51170 Köln, Germany \\ ${ }^{2}$ Institut für Planetenforschung, Deutsches Zentrum für Luft- und Raumfahrt, 51170 Köln, Germany \\ ${ }^{3}$ Technische Universität Berlin, Institut für Optik und Atomare Physik, \\ Straße des 17. Juni 135, 10623 Berlin, Germany
}

(Dated: August 19, 2021)

\begin{abstract}
Light-scattering in the terahertz region is demonstrated for granular matter. A quantum-cascade laser is used in a benchtop setup to determine the angle-dependent scattering of spherical grains as well as coffee powder and sugar grains. For the interpretation of the form factors for the scattering from single particles one has to go beyond the usual Rayleigh-Gans-Debye theory and apply calculations within Mie theory. In addition to single scattering also collective correlations can be identified and extracted as a static structure factor.

PACS numbers: 07.57.Kp,78.35.+c,81.05.Rm,42.68.Mj
\end{abstract}

The statistical physics of granular matter has seen rapid progress in recent years: In addition to macroscopic measurements, experiments with direct imaging in two dimensions 1 or tomography in three dimensions [2] allow for analyses on the scale of individual particles. Many computer-simulation studies provide testable predictions for particle packings or reveal intriguing structural anomalies [3]. Below the length scales of typical granular particles lies the realm of colloidal suspensions where light scattering has emerged as a reliable technique for the investigation of such structural properties and phase transitions [4. Scattering offers the principle advantages of (a) being able to monitor the time evolution of the measured system, (b) good resolution of both larger and smaller length scales with reliable statistics, and (c) the in-situ measurement of three-dimensional samples. Hence, the use of scattering techniques is desirable also for granular matter.

With practical laser sources now emerging in the terhertz region, light scattering with wavelengths between $30 \mu \mathrm{m}$ and $1 \mathrm{~mm}$ becomes possible [5]. As these wavelengths match typical particle sizes in granular media [6, 7], but also sizes of cells and unicellular organisms [8], suitable setups as well as methods for interpretation shall unlock the potential for terahertz light scattering in these areas. In the following, we present a bench-top light-scattering configuration using terahertz radiation, and demonstrate it for the investigation of granular particles between $80 \mu \mathrm{m}$ and $1 \mathrm{~mm}$ in diameter. By suitable interpretation of the scattering intensities, we determine the particle sizes and for high densities identify the correlations among the particle positions in a static structure factor. The results demonstrate the possibility to investigate structures on previously unattainable length scales by scattering techniques in the terhertz region.

$\mathrm{THz}$ extinction spectra obtained from samples comprising granular media have already indicated sensitivity of the extinction to particle size and packing density [912. However, such measurements are superpositions of spectroscopic features of the sampled materials as well as scattering effects, and therefore difficult to interpret. Measurement of the angular dependence of scattered intensities at a single wavelength leads to sensitivity to geometric features of the sample alone.

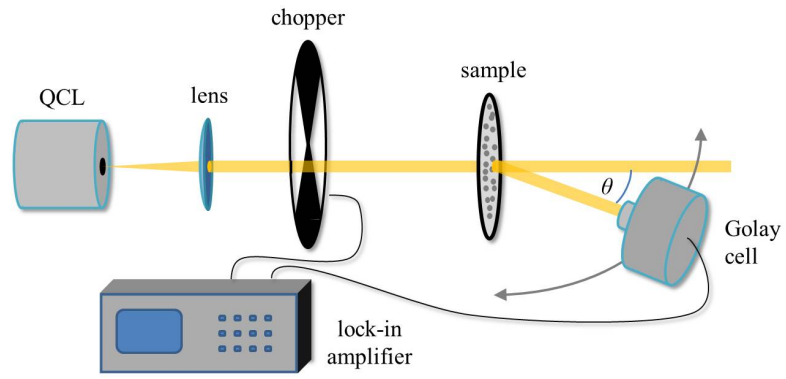

FIG. 1. Setup for $\mathrm{THz}$ scattering from granular matter. A beam from a quantum cascade laser (QCL) operating at $3.4 \mathrm{THz}$ is chopped in a lock-in configuration to suppress thermal background radiation. The incoming beam is scattered from a quasi-two dimensional granular sample at variable angle $\theta$. A Golay-cell is used as a detector.

The development of terahartz quantum cascade lasers (QCLs) 13, 14 make experiments feasible that are in close analogy to static light scattering (SLS) setups which apply visible light. In SLS, lasers provide collimated, monochromatic, high intensity radiation. Common solvents like water, organic liquids and air are transparent, and background light is easily shielded by lightproof containers. Using $\mathrm{THz}$ radiation imposes several constraints not present in experiments with visible light: Many media including ambient air are highly absorbing over large regions of the $\mathrm{THz}$ spectrum, and strong background radiation from thermal emission at room temperature has to be faced [5]. To overcome these constraints we use (1) a transmission window of air at $3.4 \mathrm{THz},(2)$ thin, effectively two-dimensional samples and (3) lock-in detection of the signal. Consequently, we obtained a versatile bench-top experiment for angle-resolved scattering 
experiments (see Fig. 11). The capabilities of the setup are demonstrated in the following to characterize spherical particles with diameters from $80 \mu \mathrm{m}$ to $1000 \mu \mathrm{m}$ as well as technical grade coffee powder and sugar grains.

First, polystyrene and polyethylene spheres are used with nominal diameters of $80 \mu \mathrm{m}$ ('PS 80'), $250 \mu \mathrm{m}$ ('PS 250'), $500 \mu \mathrm{m}$ ('PS 500'), and $1000 \mu \mathrm{m}$ ('PE 1000'). From microscopy images we obtained the particle radius $a$, the standard deviation $\sigma$ and the polydispersity $\mathrm{PD}=\sigma / a \cdot 100$ of the PS and PE particles. The size ratios $x=2 \pi \cdot a / \lambda$ and the phase shifts $\rho=2 x \cdot|m-1|$ were calculated using $a$ and literature values for the complex refractive indices relative to air of $m_{\mathrm{PS}}=1.59-i \cdot 0.002$ and $m_{\mathrm{PE}}=1.54-i \cdot 0.0014$ [15]. The number of illuminated particles followed from the cross-section of the laser beam of $3 \mathrm{~mm}^{2}$ and the packing fraction of 0.55 determined from microscopy images. The scattering vector is determined by the scattering angle $\theta$ and the wavelength in air $\lambda, q=4 \pi / \lambda \cdot \sin (\theta / 2)$. From the packing density and the beam cross section, the number of illuminated particles was estimated (see Supplemental Material for description and determination of parameters). Table I summarizes the characteristic parameters of the samples.

TABLE I. Summary of the sample characterization. $a$ is the particle radius and $\mathrm{PD}$ is the polydispersity of the particles as measured by light microscopy, $x$ the size ratio, $\rho$ the phase shift, and $n_{i l l}$ the number of particles illuminated by the primary beam.

\begin{tabular}{lccccc}
\hline \hline name & $\mathrm{a}[\mu \mathrm{m}]$ & $\mathrm{PD}[\%]$ & $x$ & $\rho$ & $n_{\text {ill }}$ \\
\hline PS 80 & 40.3 & 9.2 & 3.028 & 3.57 & $\approx 250$ \\
PS 250 & 109.5 & 5.1 & 8.327 & 9.83 & $\approx 40$ \\
PS 500 & 286.1 & 4.6 & 21.65 & 25.55 & $\approx 5$ \\
PE 1000 & 500 & n.d. & 37.85 & 40.88 & $\approx 2$ \\
\hline \hline
\end{tabular}

A custom-made QCL, obtained from the Paul-DrudeInstitut für Festkörperelektronik, Berlin, provided linearly polarized $\mathrm{THz}$ radiation with an average wavelength in air of $87.2 \mu \mathrm{m}$. We measured in $1.5^{\circ}$ steps from $-20^{\circ}$ to $100^{\circ}$ around the sample with an angular resolution of $2.6^{\circ}$ using a Golay cell. A thin PE foil supported the particles with an angle of $20^{\circ}$ to the primary beam to allow asymmetric measurements up to scattering angles of $100^{\circ}$. Background intensity measurements with empty PE foil showed scattering up to $20^{\circ}$, the fullwidth at half-maximum was determined to $14.1^{\circ}$. The obtained scattering spectra from particles were corrected and normalized for background and drift in detector response and QCL power.

The intensity of the radiation scattered by the particles exhibits variations with angle and particle size. Figure 2 shows the measured intensities for scattering angles from $\theta_{\min }=12^{\circ}$ up to $\theta_{\max }=100^{\circ}$. In a first step to interpret the measured variations, we compare the experimental data with theoretical predictions for scattering

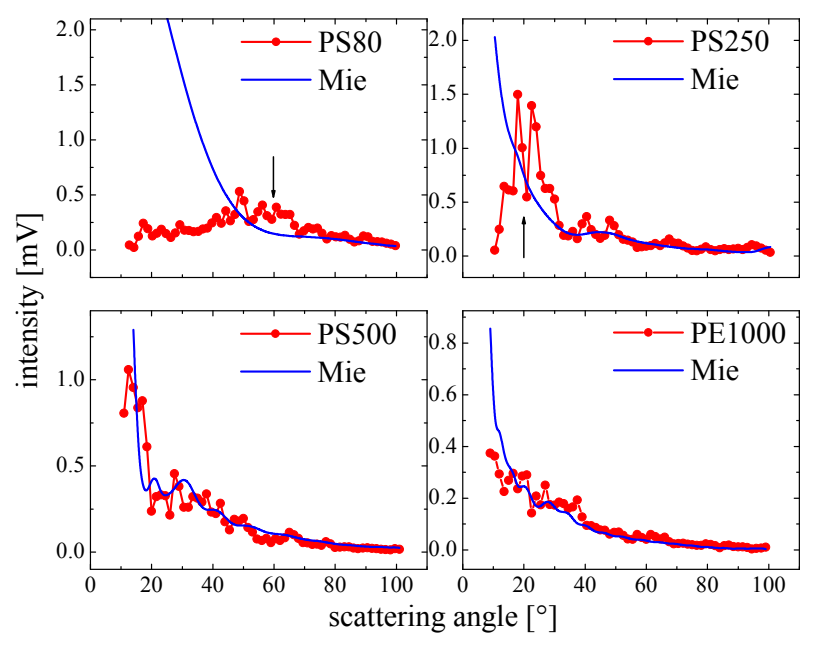

FIG. 2. Angle-resolved $\mathrm{THz}$ scattering intensities for spherical particles. Mie-theory predictions for scattering from single spheres fit the experimental background-corrected scattered intensities of polystyrene (PS) and polyethylene (PE) samples. Deviations at small angles in PS 80 and PS 250 indicate scattering from particles with correlated positions (see text). Arrows for PS 80 and PS 250 show the scattering angle below which length scales larger than the particle diameter are probed and deviations are expected.

from single spheres. From this we shall obtain quantitative information on the particle size and discuss the systematic deviations from theory in terms of correlated particle positions. The phase shifts $\rho$ produced by the particles (see Tab. I) exceeds the assumption underlying Rayleigh-Gans-Debye (RGD) theory by far: $\rho<1$ is commonly used in SLS-measurements [16], while phase shifts and size ratios larger than 1 indicate a much better applicability of the approximative theories of anomalous diffraction by van de Hulst (vdH) [17. We calculated scattered angle-dependent intensities according to RGD and $\mathrm{vdH}$ as well as Mie theory. Fitting of the data was performed using Matlab codes (www.mathworks.com). Scattering amplitudes for anomalous diffraction were calculated using the first three terms of the vdH series [17]. The Mie solution to the scattering problem was calculated using $x+4 \cdot x^{1 / 3}+2$ scattering coefficients in the Matlab-implementation by C. Mätzler [18] of the BohrenHuffman code [19] following the Wiscombe critaeria [20], cf. details in the Supplementary Material. The scattering amplitude as calculated from RGD theory depends only on the particle radius and the scattering angle. Scattering amplitudes for anomalous diffraction additionally take into account the phase shift and the full complex refractive index of the particles [17]. The polarization of the $\mathrm{THz}$ radiation perpendicular to the scattering plane is taken into account in the Mie calculations. Additionally, the divergence of the primary beam was taken into account by a moving average over scattering angles of $15^{\circ}$ (see Supplemental Material for details of the used 
models).

The agreement among the predictions of the Mie theory and the measured intensities can be seen in Fig. 2 , Fitting of the experimental scattering data with the theoretical models allows determining the particle radius. Excellent agreement can be seen in Fig. 3 between the particle radius as obtained from scattering and Mie Theory as compared to light microscopy. The vdH theory is nearly in as good agreement with the microscopy data as the Mie theory, while RGD theory systematically underestimates particle sizes.

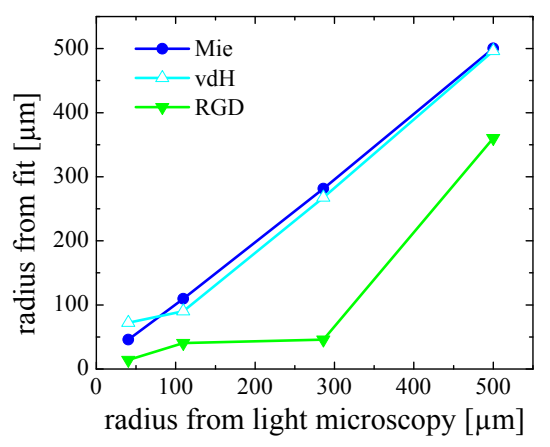

FIG. 3. Determination of the particle radius by scattering. Particle radii measured by least-square fits of Mie-theory (Mie) and predictions by the approximative theories of van de Hulst (vdH) and Rayleigh-Gans-Debye (RGD) are compared to microscopy measurements. The results from the Mie-theory are nearly indistinguishable from a bisecting line.

All applied scattering theories are strictly valid only for scattering from individual spheres with uncorrelated positions. For the smaller spheres, PS 80 and PS 250, deviations by correlations among the particle positions can be expected. The most pronounced effect of interference of light scattered from particles with correlated positions is the suppression of scattering at small scattering angles [21]. This can be observed in the scattering spectra of PS 80 and PS 250. Arrows in Fig. 2 indicate where the respective scattering angles correspond to a length scale of the particle diameter, $\sin \left(\theta^{*} / 2\right)=\lambda /(4 a)$. Smaller scattering angles, $\theta \lesssim \theta^{*}$, hence describe length scales larger than the particle diameters. The Mie prediction for single-sphere scattering alone overestimates scattered intensities at these angles. For our thin samples this suppression can be described by the structure factor $S(q)$, the Fourier-transform of the point pattern representing the particle positions [21]. The scattered intensity $I_{\text {sca }}$ becomes the product of the single-sphere scattering amplitude $P(q)$ and the structure factor $S(q)$ : $I_{\text {sca }}(q) \propto P(q) \cdot S(q)$, where $q$ is the modulus of the scattering vector.

We divide the scattered intensity by the result of the Mie prediction for scattering from a single sphere $P_{\text {Mie }}(q)$ to get an estimate of the structure factor. Figure 4 shows the result for this calculation along with the prediction

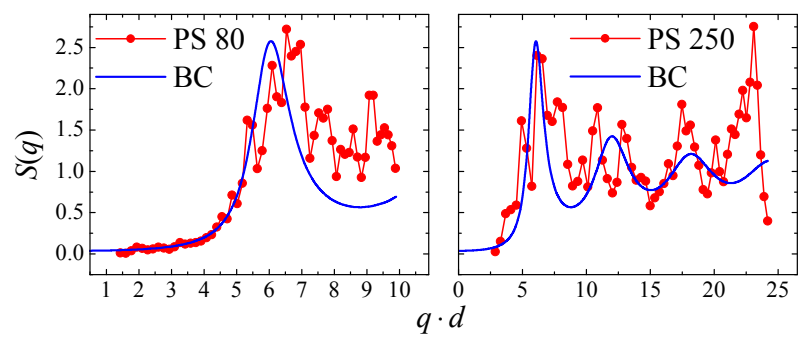

FIG. 4. Experimental structure factors from $\mathrm{THz}$ scattering. Structure factors of the samples are determined by $S(q)=$ $I_{\text {sca }}(q) / P_{\text {Mie }}(q)$ and shown together with the Baus-Colot (BC) prediction for hard-disc fluids with a packing fraction of 0.6. The scattering vector is scaled with the particle diameter.

of the Baus-Colot theory for the structure factor of twodimensional hard-disc liquids with an area fraction of 0.6 [22]. The position of the first maximum and the asymptotic behavior of the structure factor for small $q$-values agree well with the analytical prediction for hard-disc fluids. Additional fluctuations in the experimental structure factor arises from the limited number of configurations probed in the static experiments. To compare the measured structure factor with the simulation, we estimate from Fig. 2 in [3] that the predicted anomaly $S_{q} \propto q$ is found for wave vectors $q d \lesssim 3$ in the simulation. As seen in the left panel of Fig. 4, in the experiment the wave vectors for the $80 \mu \mathrm{m}$ particles start around $q d \gtrsim 1$ allowing for enough overlap to give a first estimate about $S_{q}$ in the indicated regime. The Baus-Colot fit would indicate within the experimental resolution both the absence of a linear regime in $S_{q}$ as well as the existence of a finite rather than a vanishing intercept for $q d \rightarrow 0$ at the given particle density. Given the low intensities in that respective wave-vector regime, caution is appropriate not to overstate that finding. While our results can not yet be compared with the simulation at close packing, the granular structure factors away from the transition point are consistent with the simulation [3] and show the applicability of $\mathrm{THz}$ scattering in the relevant regime of wave vectors.

To prove the applicability to technical grade particles, we demonstrate scattering from coffee powder and sugar grains in Fig. 5. We show the background-corrected scattered intensities along with the fit of Mie-scattering curves. The refractive index of polystyrene was used in the fitting procedure. The imaginary part of the refractive index had to be increased to improve fit quality. From the Mie prediction one can fit the particle radii of $300 \mu \mathrm{m}$ for coffee powder and $600 \mu \mathrm{m}$ for sugar grains, respectively. This is within expectation for the millimeter-sized sugar grains, and may indicate a sensitivity to agglomerates of the $300 \mu \mathrm{m}$ large coffee grains.

In summary, we demonstrated a versatile bench-top setup for static $\mathrm{THz}$ radiation scattering. With this setup, size-dependent angle-resolved scattered intensi- 


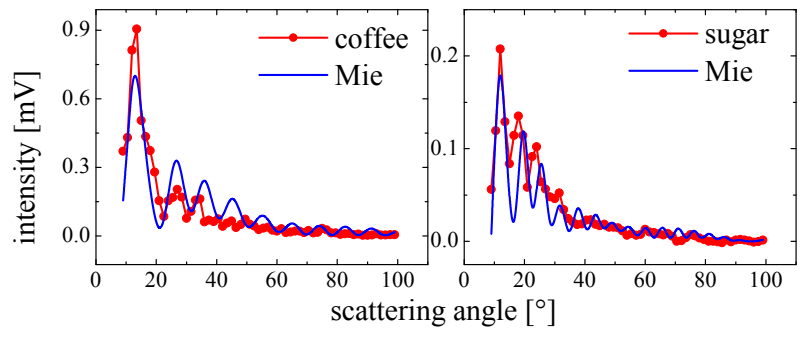

FIG. 5. Background-corrected scattered intensities of coffee powder (left) and sugar grains (right). Curves indicate leastsquare fits of Mie-theory calculations used to determine the particle sizes.

ties can be measured. By using Mie theory or the van de Hulst approximations, $\mathrm{THz}$ radiation scattering can be used to determine particle sizes. For quasi twodimensional particle packings, also static structure factors can be measured, which indicate the correlations among the particle positions. Concludingly, we have shown that by closing the gap between the infrared and the microwave part of the electromagnetic spectrum, a window opens for scattering methods to determine structural properties of particles with submillimeter sizes.

We thank H. T. Grahn, R. Hey, L. Schrottke, and M. Wienold from the Paul-Drude-Institut für Festkörperelektronik for providing the QCL. N. R. acknowledges support from the Helmholtz-Research School on Security Technologies. P. B. thanks P. Kuhn for discussions and A. Meyer for continued support.

[1] T. S. Majmudar and M. Sperl and S. Luding and R. P. Behringer, Phys. Rev. Lett. 98, 058001 (2007).

[2] M. Jerkins, M. Schröter, H. L. Swinney, T. J. Senden, M. Saadatfar, and T. Aste, Phys. Rev. Lett. 101, 018301 (2008).

[3] A. Donev, F. H. Stillinger, and S. Torquato, Phys. Rev. Lett. 95, 090604 (2005).

[4] P. N. Pusey and W. van Megen, Nature 320, 340 (1986).

[5] E. Bündermann, H.-W. Hübers, and M. Kimmitt, Terahertz Techniques. (Springer, 2012).

[6] M. J. Rhodes (Ed.), Principles of Powder Technology. (John Wiley \& Sons, 1991).

[7] J. Duran, Sands, Powders, and Grains: An Introduction to the Physics of Granular Materials. (Springer, 1999).

[8] N. A. Campbell (Ed.), Biology: Exploring Life. (Pearson Prentice Hall, 2003).

[9] L. M. Zurk, B. Orlowski, D. P. Winebrenner, E. I. Thorsos, M. Leahy-Hoppa, and M. R. Hayden, J. Opt. Soc. Am. B 24, 2238 (2007).

[10] A. Bandyopadhyay, A. Sengupta, R. B. Barat, D. E. Gary, J. F. Federici, M. Chen, and D. B. Tanner, J Infrared Milli Terahz Waves 28, 969 (2007).

[11] M. Kaushik, B. W.-H. Ng, B. M. Fischer, and D. Abbott, Appl. Phys. Lett. 100, 011107 (2010).

[12] M. Kaushik, B. W.-H. Ng, B. M. Fischer, and D. Abbott,
Appl. Phys. Lett. 100, 241110 (2012).

[13] R. Köhler, A. Tredicucci, F. Beltram, H. E. Beere, E. H. Linfield, A. G. Davies, D. A. Ritchie, R. C. Iotti, and F. Rossi, Nature 417, 156 (2002).

[14] H. Richter, M. Greiner-Bär, S. G. Pavlov, A. D. Semenov, M. Wienold, L. Schrottke, M. Giehler, R. Hey, H. T. Grahn, and H.-W. Hübers, Opt. Express 18, 10177 (2010).

[15] P. D. Cunningham, N. N. Valdes, F. A. Vallejo, L. M. Hayden, B. Polishak, X.-H. Zhou, J. Luo, A. K.-Y. Jen, J. C. Williams, and R. J. Twieg, J. Appl. Phys. 109, 043505 (2011).

[16] S. U. Egelhaaf, Solution Scattering. In Soft condensed matter physics in molecular and cell biology, ed. by W. C. K. Poon and D. Andelman, p. 38. (Taylor \& Francis, 2006).

[17] H. C. van de Hulst, Light Scattering by Small Particles. (Dover Publications, 1981).

[18] C. Mätzler, MATLAB Functions for Mie Scattering and Absorption. IAP Research Report, Institut für angewandte Physik, Universität Bern 08, (2002).

[19] C. F. Bohren and D. R. Huffman, Absorption and Scattering of Light by Small Particles. (Wiley-VCH, 1983).

[20] W. J. Wiscombe, Appl. Opt. 19, 1505 (1980).

[21] W. Brown (Ed.), Light scattering: principles and development. (Clarendon Press, 1996).

[22] M. Baus and J. L. Colot, J. Phys. C 19, L643 (1986). 


\section{SUPPLEMENTAL MATERIAL}

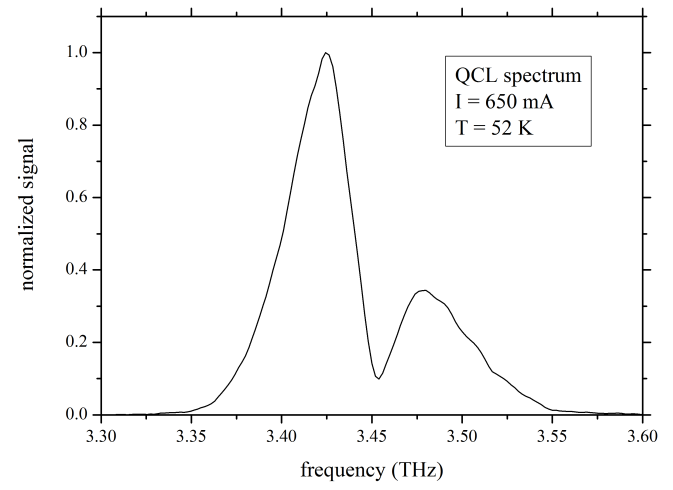

FIG. 6. Spectrum of the radiation emitted by the quantum cascade laser at $52 \mathrm{~K}$. Two modes are observable, the peak heights are used to determine the average wavelength of the laser in air.

Setup A custom-made quantum cascade laser (QCL), obtained from the Paul-Drude-Institut für Festkörperelektronik [14, Berlin, provided the required radiation. At $52 \mathrm{~K}$, the laser emitted approximatively $2.2 \mathrm{~mW}$ in two modes at $3.42 \mathrm{THz}(87.55 \mu \mathrm{m})$ and $3.48 \mathrm{THz}(86.15 \mu \mathrm{m})$ linearly polarized perpendicular to the scattering plane. An emission spectrum can be found in Fig. 6. From the peak heights an average frequency of $3.44 \mathrm{THz}(87.2 \mu \mathrm{m})$ was determined. A plano-convex TPX lens was adjusted $90 \mathrm{~mm}$ from the laser in the light path to minimize beam divergence. Measurements with a modified mid-IR microbolometer camera (Infratec Variocam) gave a beam cross-section of $3 \mathrm{~mm}^{2}$ at the sample position (see Figs. 7 and 8 .

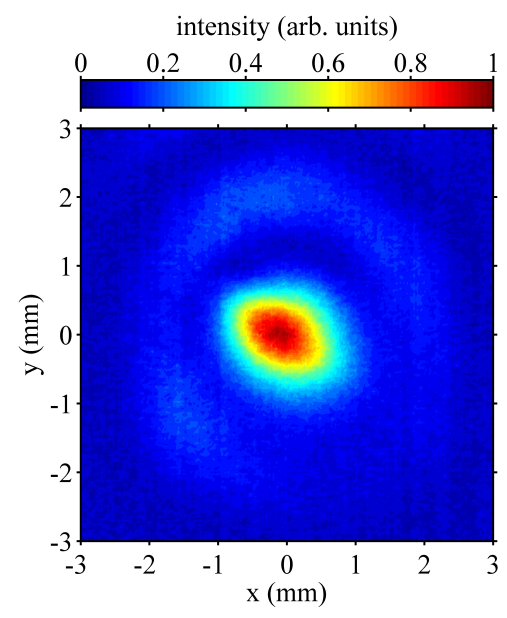

FIG. 7. Intensity profile of the laser beam. At the position of the sample the beam has a nearly Gaussian shape.

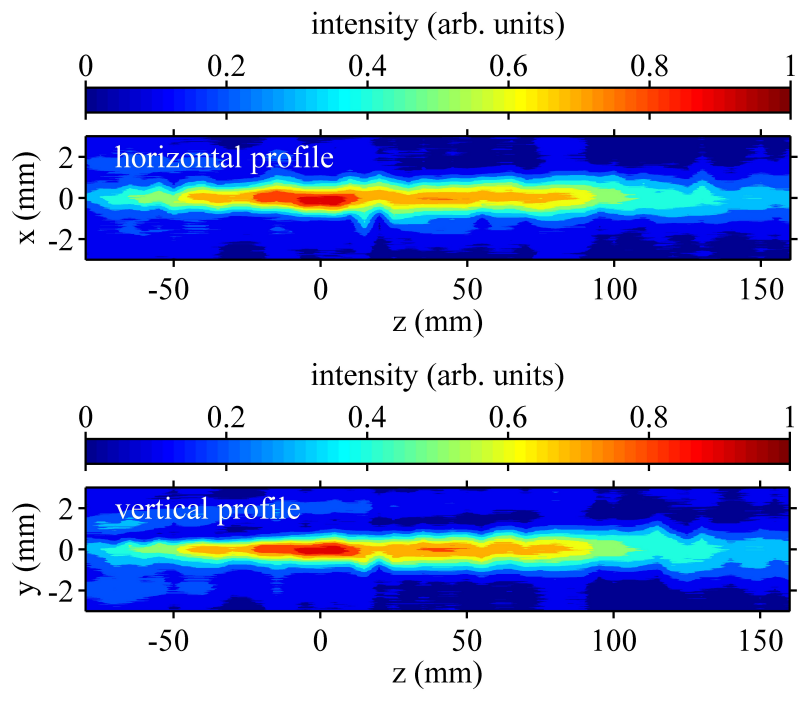

FIG. 8. Profile of the laser beam in the scattering plane (top) and in the plane perpendicular to the scattering plane (bottom). The sample is located at $z=0$.

A goniometer (PI M-660.55 rotation stage) was installed $600 \mathrm{~mm}$ from the lens for angle-resolved measurements. A Golay-cell (Tydex GC-1T) measured the light intensity on an extension arm $55 \mathrm{~mm}$ from the center of the goniometer. That led to a total free path length in air of $745 \mathrm{~mm}$. A $2.5 \mathrm{~mm}$ wide circular diaphragm in front of the cell entrance window ensured an angular resolution of $2.6^{\circ}$.

The application of a Golay-cell as detector and the strong thermal background made chopping the light signal and lock-in detection necessary. We chopped the light with $10 \mathrm{~Hz}$ and detected with $2 \mathrm{mV}$ lock-in sensitivity (Stanford Research SR 850 amplifier). This led to saturation of the detector signal in the primary beam but ensured sensitivity to weak scattering signals.

A very thin sample holder was necessary to minimize absorption losses. $10 \mu \mathrm{m}$ thin polyethylene (PE) foil was tautened over a metal ring with $7.5 \mathrm{~cm}$ diameter. By pressing and removing adhesive tape onto and from the foil a thin residual adhesive film was created. The sample holder took an angle of $20^{\circ}$ with respect to the laser beam to allow asymmetric measurements up to high scattering angles of $100^{\circ}$ without shadowing by the metal ring. A photograph of the assembled setup is available in Fig. 9 .

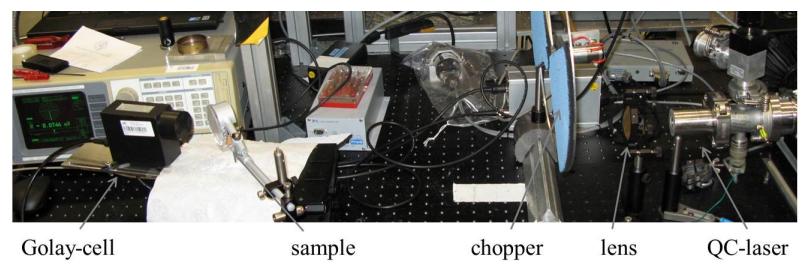

FIG. 9. Photograph of the benchtop experimental setup. 
Samples Polystyrene (Dynoseeds, Microbeads AS) and polyethylene spheres (Sphero, Rieter $\mathrm{GmbH}$ ) were used as purchased. Light microscopy images were taken of the polystyrene samples and evaluated using ImageJ (http://rsbweb.nih.gov/ij/). The mean particle size and the size distribution was determined from the light microscopy images (Fig. 10.

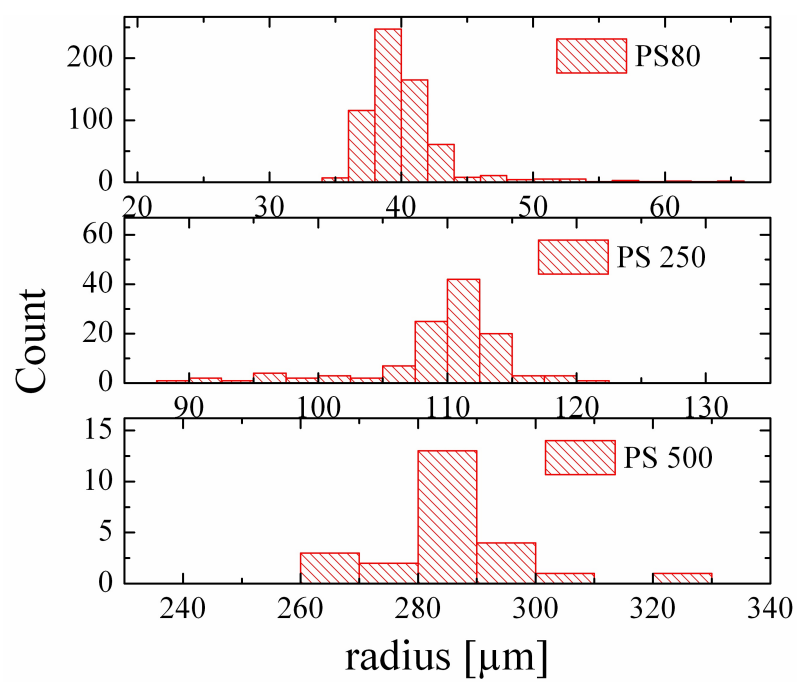

FIG. 10. Size distribution of the polystyrene samples as measured with light microscopy.

The polydispersity PD was defined by the standard deviation $\sigma$ of the mean radius $a$ and the mean radius $a$, $\mathrm{PD}=\sigma / a \cdot 100$. The particles were spread on the adhesive film on the $\mathrm{PE}$ foil; subsequently unfixed particles were blown off, yielding a packing density of deposited $\phi \approx 55 \%$ (see Fig. 11 for a microscopy image of the deposited particles). For calculation of $x$ and $\rho$ literature values for the complex refractive indices of PS and PE were used, $m_{P S}=1.59-i \cdot 0.002$ and $m_{P E}=1.54-i \cdot 0.0014$ 15

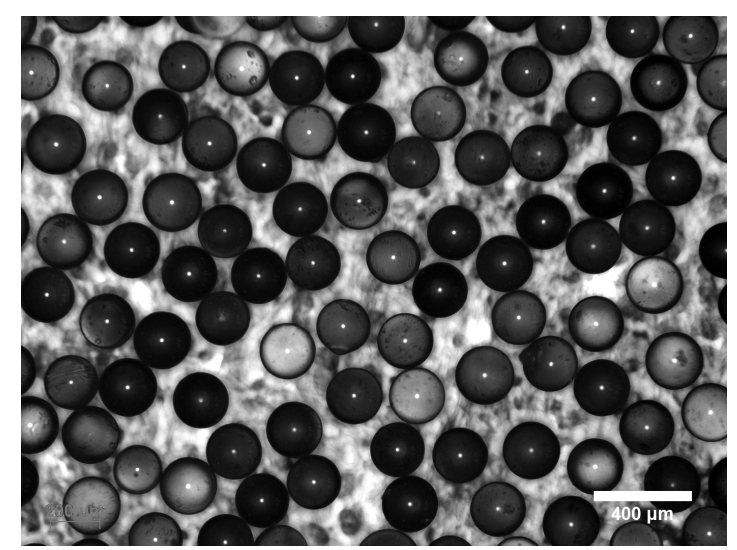

FIG. 11. Representative image of the deposited granular particle samples, here PS 250.
Size-dependent angle-resolved scattering Measurements were performed from $-20^{\circ}$ to $100^{\circ}$ in $1.5^{\circ}$-steps. The intensity scattered by the PE foil and the adhesive film was measured prior to particle measurements. The beam exhibited scattering up to $20^{\circ}$, the full-width halfmaximum was determined to $14.1^{\circ}$. Above $20^{\circ}$ virtually no scattered intensity was detected (see Fig. 12). The scattering spectra from the particles were corrected and normalized for the background from the empty sample holder and the drift in detector response and QCL power.

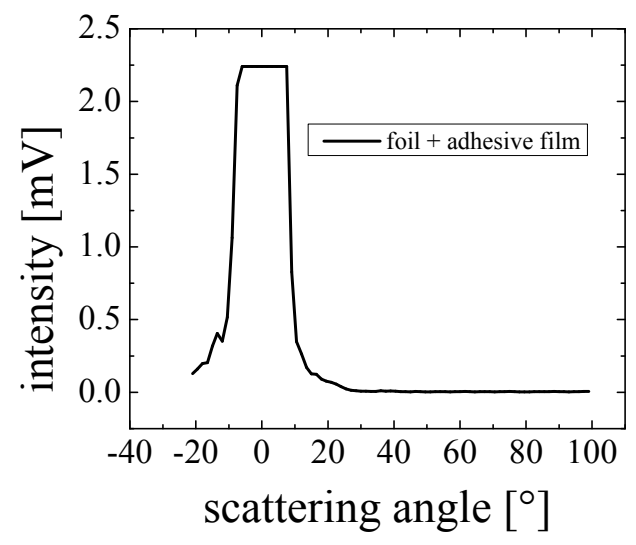

FIG. 12. Scattering spectrum of the laser beam after it passed through the PE foil. The signal in the range from $-10^{\circ}$ to $10^{\circ}$ is saturated, a Gaussian fit yields a full-width half maximum of $14.1^{\circ}$, above $20^{\circ}$ no scattered intensity is detectable.

Fitting Fitting of the data was performed using Matlab codes and the least-square fitting routines provided by the optimization toolbox for Matlab (www.mathworks.com). A scaling factor and the particle radius was left as a free fit parameter. Three models for single sphere scattering were tested: RayleighGans-Debye theory (RGD), van de Hulst's approximation (vdH), and the exact Mie-solution (Mie). RGD theory provides a form factor $P_{\mathrm{RGD}}(q)$ for scattering from a single sphere [17, which depends only on the particle radius $a$ and the scattering vector $q=4 \pi / \lambda \cdot \sin (\theta / 2)$ :

$$
P_{\mathrm{RGD}}(q, a)=\left[\frac{3}{(q a)^{3}}(\sin (q a)-q a \cos (q a))\right]^{2} .
$$

RGD theory can be expected to give meaningful results only for particles with $\rho \ll 1$. The fit quality using RGD theory was generally poor, we obtained root-meansquare(rms)-deviations of $6.5 \mathrm{mV}(23.7 \%$, averaged over all particle sizes).

Van de Hulst dropped the limitation of $\rho$ being small and obtained a series expansion for the scattering from individual spheres [17, albeit still with the restriction of the refractive index $m$ being close to unity, $|m-1|<1$. The series converges with $1 / \rho^{2}$; we used the first three terms of the series expansion. Using the next higher term in the series yielded only relative changes of the scatter- 
ing amplitude on the order of $10^{-2}$. The scattering amplitude in the $\mathrm{vdH}$ approximation takes the full complex refractive index into account:

$$
\begin{aligned}
P_{\mathrm{vdH}}(m, a, \theta)= & {\left[\frac{\rho}{y^{2}}\left(\frac{\pi y}{2}\right)^{1 / 2} J_{3 / 2}(y)\right]^{2} } \\
& +\left[\frac{1}{z} J_{1}(z)+\frac{\rho}{y^{2}}\left(\frac{\pi y}{2}\right)^{1 / 2} Y_{3 / 2}(y)\right. \\
& \left.+\frac{1}{\rho^{2}} J_{0}(z)+\ldots\right]^{2},
\end{aligned}
$$

where $z=x \cdot \theta, y^{2}=\rho^{2}+z^{2}$, and $J_{\alpha}(y)$ and $Y_{\alpha}(y)$ being Bessel functions of the first and the second kind, respectively. The fit quality using $\mathrm{vdH}$ theory was very good, we obtained $\mathrm{rms}$ deviations of $0.4 \mathrm{mV}(7.7 \%$, averaged over all particle sizes).

To calculate the exact Mie-theory solution to the scattering problem the Matlab-implementation of the Bohren-Huffman code [19] by C. Mätzler was used [18]. Following the criteria given by Wiscombe, $x+4 \cdot x^{1 / 3}+2$ scattering coefficients were calculated to obtain the scattering amplitude 20. The polarization of the $\mathrm{THz}$ radiation perpendicular to the scattering plane is taken into account in the Mie-calculations. The fit quality using Mie theory was excellent, we obtained rms-values of $0.2 \mathrm{mV}$ (4.7\%, averaged over all particle sizes).

The full-width half-maximum of $14.1^{\circ}$ of the primary beam without particles was taken into account in the comparison of the experimental scattering data to the theoretical predictions by a moving average over 10 data points or $15^{\circ}$, respectively.

Structure factor calculation The structure factor represents the phase shift between the light scattered by the individual particles; it is thus equivalent to the Fourier transform of the pair distribution function $g(r)$, the probability of finding at a distance $r$ from a first particle center a second particle center. While one cannot expect the calculations for thermal systems to hold also for static granular packings, the structure factors show generic features and trends like their principal peaks increasing with density that are also observed for packings.

Following Ornstein-Zernike, the structure factors for hard discs can be expressed as $S(q, \eta)=1 /(1-$ $4 \eta / \pi c(q, \eta))$, where $c(q, \eta)$ is the Fourier transform of a direct correlation function $c(r, \eta), r$ the particle distance scaled by the diameter and $\eta$ the area fraction. M. Baus and J.-L. Colot 22] have proposed an analytical formulation for the direct correlation function which yields typically good agreement with experiments especially in two dimensions,

$$
c(r, \eta)=\Theta(1-r) c(r=0, \eta)\left(1-a^{2} \eta(1+\omega(r / a)),\right.
$$

where $\Theta$ is the Heavyside function, $\omega(x)=$ $(2 / \pi)\left[\cos ^{-1} x-x\left(1-x^{2}\right)^{1 / 2}\right]$ and $a$ is a scaling factor derived from thermodynamic arguments. We adjusted the area fraction to match the theoretical prediction for $S(q, \eta)$ with the experimental data. 